\title{
Type of feature affects transfer in operant serial feature-positive discriminations
}

\author{
MURRAY J. GODDARD \\ University of New Brunswick, Saint John, New Brunswick, Canada \\ and \\ PETER C. HOLLAND \\ Duke University, Durham, North Carolina
}

\begin{abstract}
In two experiments, the effects of feature identity in operant serial feature-positive discriminations were examined with rats. Rats were trained with two serial feature-positive discriminations $(\mathrm{F} 1 \rightarrow \mathrm{T} 1+/ \mathrm{T} 1-$ and $\mathrm{F} 2 \rightarrow \mathrm{T} 2+/ \mathrm{T} 2-)$, in which different operants were reinforced with delivery of a sucrose solution during two auditory target cues (T1 and T2). The features (F1 and F2) were two visual cues, two flavored sucrose solutions, or one visual cue and one sucrose solution. Transfer of a feature's control to the target of the other discrimination was observed only when the features were from the same modality. When observed, transfer responding was of the form originally trained to the target, rather than the feature, and was preserved after feature extinction. Control groups showed that the differential transfer was not solely the consequence of differential feature generalization. Implications for theories of occasion setting are discussed.
\end{abstract}

Considerable evidence has shown that reinforcers, or unconditioned stimuli (USs), can come to acquire signaling or discriminative stimulus properties in addition to their reinforcing properties (e.g., Capaldi, 1967; Goddard, Holland, O'Brien, \& Hansen, 1994; Goddard \& Jenkins, 1988; Holz \& Azrin, 1961; Staddon \& Innis, 1969). For example, Holland and Forbes (1982), using a preparatory-releaser procedure (Konorski \& Lawicka, 1959; Terry \& Wagner, 1975) with rats, paired a tone with food on some trials and presented the tone without food on other trials. In feature-positive training, the tone was paired with food only when it was preceded by a sucrose feature; in feature-negative training, the tone was paired with food only when it was not preceded by a sucrose feature. In both cases, the rats rapidly learned to respond appropriately to the tone.

Typically, results from the preparatory-releaser procedure are similar to the results from comparable experiments in which more neutral conditioned stimuli (CSs) are used as features. However, some outcomes differ, at least superficially. For example, although most investigations of feature-positive and feature-negative training find superior feature-positive performance with CS features, both Reberg and Memmott (1979) and Holland and Forbes

This research was conducted while M.J.G. was on sabbatical leave at Duke University. It was supported in part by a grant from the National Science Foundation to P.C.H. and by a Natural Sciences and Engineering Research Council of Canada grant to M.J.G. Portions of these data were reported at the annual meeting of the Psychonomic Society, St. Louis, Missouri, November 1994. Requests for reprints should be sent to M. J. Goddard, Psychology Department, UNBSJ, Saint John, New Brunswick, Canada, E2L 4L5, or P. C. Holland, Box 90086, Duke University, Durham, NC 27708-0086 (e-mail: goddard@) unbsj.ca or pch@acpub.duke.edu).
(1982) found superior feature-negative performance with US features (but see Bottjer \& Hearst, 1979). It is possible that highly salient, biologically meaningful events possess attributes or generate consequences that may alter their cuing properties, relative to less significant events. Indeed, some theorists have suggested that learning of relations among USs may differ qualitatively from CS-US learning (e.g., Garcia, 1989; Solomon, 1977).

Nevertheless, there has been little explicit comparison of learning involving relatively neutral cues with learning involving cues that are themselves reinforcers. The experiments reported here compared the functions of visual and sucrose features in operant serial feature-positive (SFP) discriminations $(\mathrm{F} \rightarrow \mathrm{T}+, \mathrm{T}-$ ) with rats. Operant responding during an auditory target was reinforced when that target was preceded by either a visual or sucrose feature; responding during the auditory target alone was not reinforced.

Holland (1983, 1992, 1995a, 1995b) claimed that operant serial feature $\rightarrow$ target relations generate learning in which the feature serves to modulate, or set the occasion for, target responding. This differs from operant simultaneous feature-target relations in which learning is based on simple feature-US associations. Several kinds of evidence support this claim. First, patterns of transfer differ after operant serial and simultaneous featurepositive discrimination training (Holland, 1991a, 1995b). Although a simultaneously trained feature controls responding regardless of whether it is presented alone, with its original target, or in compound with other, separately trained discriminative stimuli, transfer of a serially trained feature's powers is more circumscribed.

Second, after subjects are trained to emit different operants during each of two feature-positive discrimina- 
tions, the response shown in transfer tests depends on whether the compounds were trained simultaneously or serially. A simultaneously trained feature controls the same response in transfer tests that it controlled in its original compound, whereas a serially trained feature enhances the response controlled by the target cue (Holland, 1995b). Finally, although feature extinction substantially eliminates the ability of simultaneously trained features to control responding in their original training compounds, it has little effect on the ability of serially trained features to control responding in their original training compounds.

In the experiments reported here, SFP learning was compared with a light ("CS") or sucrose ("US") feature. Although the light feature was trained as an occasion setter, rather than as a CS, and sucrose can itself serve as a $\mathrm{CS}$ in conditioning experiments (for example, in taste aversion), the terms CS and US were used to simplify discussion. In Experiment 1, a within-subject design was used to compare the acquisition of one SFP discrimination using a CS feature and one SFP discrimination using a US feature. Furthermore, the extent and nature (response form) of transfer and the effect of CS or US extinction on the original and transfer discrimination performance was examined. In Experiment 2, a mixed design was used to compare performance when the two SFP discriminations involved two US features, two CS features, or one US and one CS feature, and added control procedures to evaluate the contribution of feature generalization to the observed outcomes.

\section{EXPERIMENT 1}

In Experiment 1, a single group of rats received one SFP discrimination with a CS feature and one SFP discrimination with a US feature. Leverpressing was reinforced in the presence of an auditory target when that cue was preceded by sucrose, and chainpulling was reinforced in the presence of another auditory target when it was preceded by a light. No reinforcement was available when the auditory targets were presented alone. The ability of the sucrose and light features to modulate responding to their original targets and to the target of the other SFP discrimination was then examined in a transfer test. Finally, one of the features was extinguished and the effects of feature extinction evaluated in another transfer test.

\section{Method}

Subjects. The subjects were 1 male and 9 female SpragueDawley rats, bred from Charles River (Raleigh, NC) stock in a Duke University Department of Psychology: Experimental facility. At the beginning of the experiment, the rats were about 150 days old and experimentally naive. They were maintained at $85 \%$ of their ad-lib body weights by measured feedings at the end of a session. Water was available at all times in their individual home cages.

Apparatus. There were eight identical chambers, each $22.9 \times$ $20.3 \times 20.3 \mathrm{~cm}$. The front and back walls of each chamber were aluminum; the side walls and top were clear acrylic. The floor of the chamber was composed of $0.48-\mathrm{cm}$ stainless steel rods spaced
$1.9 \mathrm{~cm}$ apart. A food cup was recessed behind a $5 \times 5 \mathrm{~cm}$ opening in the front wall; the bottom of the opening was $2 \mathrm{~cm}$ from the floor, and its center was $2 \mathrm{~cm}$ to the right of the center of the front wall. The sucrose was contained in a plastic dispenser behind the front wall. When a solenoid valve was activated, there was an audible click and approximately $0.3 \mathrm{ml}$ of $0.2 \mathrm{M}$ sucrose were delivered into the food cup. A 6-W jeweled lamp (panel light) was centered on the front wall $4 \mathrm{~cm}$ above the top of the food cup opening. Except when the panel light was illuminated as a signal, the chambers were dark. A $2 \times 2 \mathrm{~cm}$ lever was mounted $3 \mathrm{~cm}$ above the floor, $4 \mathrm{~cm}$ to the left of the food opening. A chain was suspended from the ceiling, in line with the center of the lever but $5 \mathrm{~cm}$ from the front wall.

Each of the chambers was enclosed in a sound-attenuating shell. A speaker for delivering the auditory CSs was mounted on one wall of each shell, level with the top of the chamber and $2 \mathrm{~cm}$ in front of and $10 \mathrm{~cm}$ to the left of the front wall of the chamber. Finally, each shell was enclosed in another sound-attenuating box. Constant background noise ( $68 \mathrm{~dB}$ ) was provided by a ventilating fan on each box.

Procedure. The rats were first trained to drink sucrose, press the lever, and pull the chain. During the first two sessions, only the lever was available. In the first session, the rats received 20 sucrose deliveries on a variable-time 2 -min schedule. In addition, during that $40-\mathrm{min}$ period and during the remaining $20 \mathrm{~min}$ of the session, each leverpress was reinforced. In the second session, sucrose was delivered only after each leverpress; each rat was removed after making approximately 50 leverpresses. During the third session, only the chain was available; each chainpull was reinforced, and each rat was removed after making approximately 50 chainpulls.

The next 11 sessions were designed to place leverpressing and chainpulling under the control of the targets to be used later in SFP discrimination training. For half of the rats, each leverpress was reinforced during a $72-\mathrm{dB}$ (A) square-wave $1500-\mathrm{Hz}$ tone and each chainpull was reinforced during a $72-\mathrm{dB}$ white noise. For the other half of the rats, the contingencies were reversed. In all sessions, tone and noise presentations were randomly intermixed. The first two sessions were $30 \mathrm{~min}$ long and contained fifteen 30 -sec tone and fifteen $30-\mathrm{sec}$ noise presentations. The tone and noise cues were then gradually shortened from 30 to $15 \mathrm{sec}$ (Sessions 3 and 4), $10 \mathrm{sec}$ (Sessions 5 and 6), and $5 \mathrm{sec}$ (Session 7). The session length was extended to $60 \mathrm{~min}$ in Session 8; finally, Sessions 9-11 were $64 \mathrm{~min}$ long and included eight 5 -sec tone and eight 5-sec noise presentations.

In the next 23 sessions, each rat was trained on two SFP discriminations. In one discrimination, the delivery of sucrose (US) was the feature, an auditory cue (tone or noise, counterbalanced) was the target ( $\mathrm{T} 1$ ), and leverpressing was the reinforced response. In the other discrimination, the panel light (CS) was the feature, the other auditory cue was the target (T2), and chainpulling was the reinforced response. Each 64-min session included four US-T1 + trials (each leverpress during T1 was reinforced), four $\mathrm{T} 1-$ trials (no reinforcement was available), four CS-T2+ trials (each chainpull during T2 was reinforced), and four T2trials. On CS-T2 + trials, a 20 -sec empty interval separated the termination of the panel light and the onset of $\mathrm{T} 2$. In the first two sessions of this phase, all cues were $5 \mathrm{sec}$ in duration (except for US delivery, which was $0.5 \mathrm{sec}$ ), and on US-T I + trials a $25-\mathrm{sec}$ empty interval separated the termination of the sucrose feature and the onset of $\mathrm{T} 1$. However, in the last 21 sessions, the empty interval on US-T $1+$ trials was changed from 25 to $20 \mathrm{sec}$, and all cues (except the US) were extended from 5 to $10 \mathrm{sec}$ in duration. The stimuli were extended to provide more opportunity for the rats to respond during the reinforced target cues, and the US-T2+ gap was changed to equate it with the gap on CS-T1 + trials rather than to equate the interval between the onset of a feature and the onset of a target. 
Next, the rats received two test sessions to evaluate transfer. In each 64-min session, there were two presentations of each of eight trial types. The eight trial types included the two features alone (CS and US), the two targets alone (T1 and T2), the two original serial compounds (US-T1 and CS-T2), and, of special interest, the two novel transfer compounds (US-T2 and CS-T1). Responding was not reinforced during the two test sessions.

The rats then received seven sessions in which one feature was extinguished while the other feature-target discrimination was retrained. In each 64-min session, half of the rats received eight USalone trials, four CS-T2 + trials, and four T2 - trials, whereas the other half of the rats received eight CS-alone trials, four US-T1+ trials, and four $\mathrm{T} 1$ - trials.

Finally, all subjects received two postextinction test sessions identical to the test sessions described previously.

Data analysis. We recorded the percentage of trials on which at least one response occurred during a cue, the response rates during that cue, and the latency to the first response to that cue; in addition, we recorded those same measures for a 10-sec interval before the cues (pre-CS interval). Response rate was the primary index of performance in the test sessions when reinforcement was unavailable. However, response rate was inappropriate during discrimination training when reinforcement was available. Typically, rats ceased responding immediately after a reinforced response and collected the available sucrose. Because the response rates on reinforced trials were therefore artificially low, the percentages of trials on which at least one response occurred were reported during discrimination training. The latency data were similar to the rate and percentage data, and are not presented here.

The choice of target cues (tone or noise) did not affect responding in any data set analyzed, so the data were collapsed across those factors. Nonparametric inferential statistics were used, with a criterion of $p \leq .05$, two-tailed.

\section{Results}

During initial training, the rats learned to perform one response during one target cue and a different response during the other target cue. Leverpressing was more readily differentiated than chainpulling; over the first 10 sessions, the difference between the percentage of trials on which leverpressing was performed when it was the correct, reinforced response and when it was the incorrect, nonreinforced response was greater than the analogous difference for chainpulling [Wilcoxon $T(10)=0$ ]. However, by the final session of this phase, the two responses were equally well differentiated $[T(8,2$ ties $)=11]$. In that final session, on trials when leverpressing was the correct response, the rats made at least one correct response on $65 \%$ of trials and at least one incorrect response on $19 \%$ of trials; when chainpulling was the correct response, the rats made at least one correct response on $60 \%$ of trials and at least one incorrect response on $25 \%$ of trials.

Figure 1 shows performance of target-appropriate responding in the last 21 sessions of discrimination training, after the feature-target gaps were equated (responding inappropriate to the target occurred on fewer than $7.5 \%$ of the trials, and is not shown). The two SFP discriminations were acquired at similar rates: the discrimination difference scores (appropriate responding on compound trials minus that on target-alone trials) did not differ reliably $[T(10)=13]$.

Figure 2 shows responding during the first two test sessions. Performance on the two original discriminations

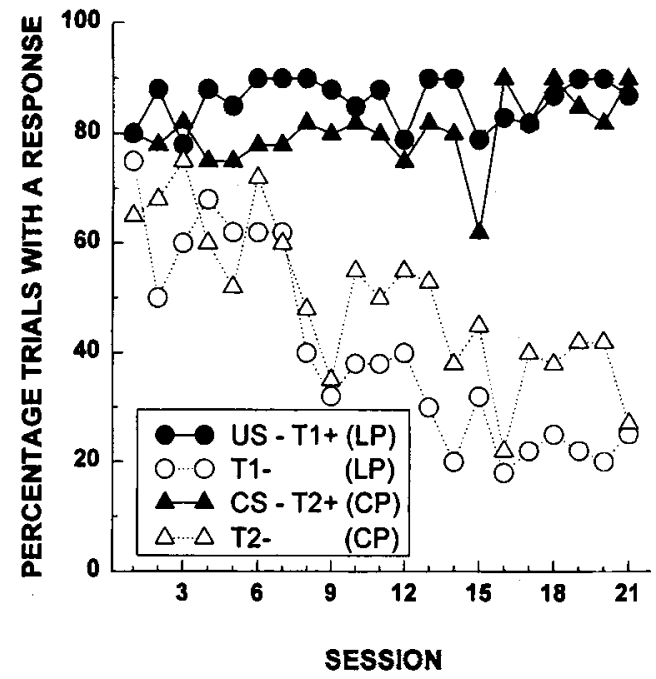

Figure 1. Percentage of trials on which an appropriate response (LP, leverpress, or CP, chainpull) occurred during the target cues (T1 and T2) during acquisition of the two serial feature-positive discriminations in Experiment 1. Data from two sessions prior to the adjustment of conditioning parameters (see text) are not shown. $+=$ serial compound trials on trials on which reinforcement was available; $-=$ target-alone trials on which reinforcement was not available; $U S=$ sucrose feature; $C S=$ visual feature.

was maintained: leverpressing was significantly greater during US-T1 trials than during T1 trials, and chainpulling was reliably greater on CS-T2 trials than on $\mathrm{T} 2$ trials $[T(9)=1.5]$. In addition, there was significantly more leverpressing on US-T1 trials than on US-alone trials $[T(9)=1]$ and reliably more chainpulling on CS-T2 trials than on CS-alone trials $[T(10)=7]$. This higher responding on compound trials than on feature-alone trials suggests that responding to the original compounds may have been based, at least partly, on occasion setting rather than on simple feature conditioning.

Of greatest interest, however, was the responding to the two novel transfer compounds (US-T2 and CS-T1).

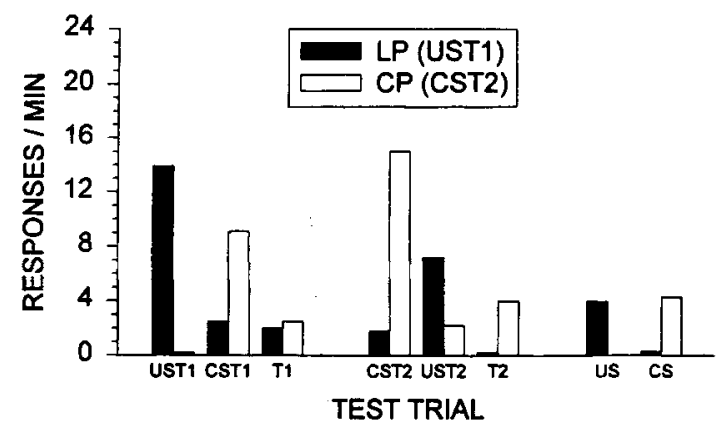

Figure 2. Mean response rates during the first transfer test of Experiment 1. In training, leverpressing (LP) was reinforced on US-T1 compound trials, and chainpulling (CP) was reinforced on CS-T2 compound trials. Responding on feature-alone trials (labeled US and CS) was measured at the time when a target would normally have been presented. $U S=$ sucrose feature; $C S=$ visual feature. 
Transfer occurred, but was attributable to feature conditioning: responding to each compound was dominated by feature-specific responding and did not differ from responding observed on feature-alone trials. Leverpressing (originally trained with the US-T1 compound) was reliably more frequent than chainpulling on US-T2 trials $[T(9)=6]$, and chainpulling (originally trained with the CS-T2 compound) was reliably more frequent than leverpressing on CS-T1 trials $[T(8)=0]$. Feature-specific leverpressing was significantly more frequent to the US$\mathrm{T} 2$ compound than to $\mathrm{T} 2$ alone $[T(8)=0]$, and chainpulling was numerically (but not reliably) more frequent to the CS-T1 compound than to $\mathrm{T} 1$ alone $[T(10)=9.5$, $.05<p<.10$ ]. In contrast, neither of the target-specific responses was significantly more frequent on transfer compound trials than on target-alone trials $\left(T_{\mathrm{s}} \geq 20\right)$. Finally, responding to the transfer compounds was not reliably greater than responding to the features alone: there was no more leverpressing on US-T2 compound trials than on US-alone trials $[T(10)=17]$ or more chainpulling on CS-T1 trials than on CS-alone trials $[T(7)=12]$.

Figure 3 shows responding during identical transfer tests that were administered after US (top panel) or CS (bottom panel) feature extinction. In general, feature extinction resulted in a loss of responding on feature-alone trials and on transfer compound trials with that feature, whereas responding to the original feature-target compound was maintained. Because the results after US and CS extinction were qualitatively similar, the two subgroups were combined for statistical analysis. Compared
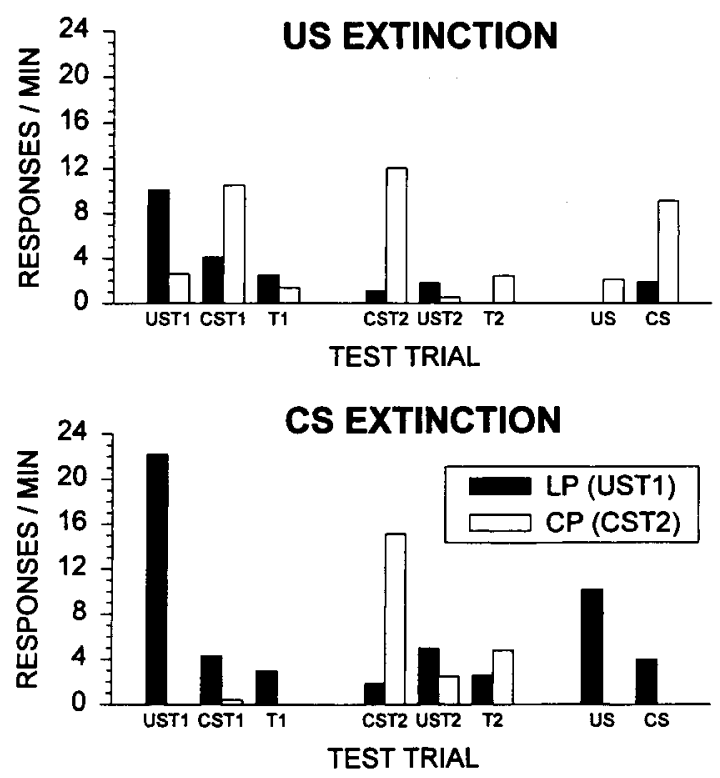

Figure 3. Mean response rates during the second transfer test of Experiment 1, after either US (top panel) or CS (bottom panel) feature extinction. In training, leverpressing (LP) was reinforced on US-T1 compound trials, and chainpulling was reinforced on CS-T2 compound trials. Responding on feature-alone trials (labeled US and CS) was measured at the time when a target would normally have been presented. $U S=$ sucrose feature; $C S=$ visual feature. with responding in the identical tests administered before feature extinction, there was a significant loss in responding to the feature $[T(9)=0]$ and transfer compound $[T(8)=2]$ but no reliable loss in responding to the original compound $[T(10)=19]$. Therefore, it is likely that responding to the novel transfer compounds was based primarily on feature conditioning whereas responding to the original feature-target compounds was based, at least partly, on occasion setting.

Unexpectedly, responding on trials with the nonextinguished feature significantly increased after extinction of the other feature ( $T \mathrm{~s}=0$ for feature-alone and transfer trials and $T(9)=5.5$ for original compound trials). It is unclear whether these increases reflected some sort of contrast effect, additional training on the nonextinguished discrimination, or some other factor.

\section{Discussion}

In Experiment 1, all subjects received one SFP discrimination with a CS feature and one SFP discrimination with a US feature. Both discriminations were learned at comparable rates. The results showed that simple feature conditioning contributed to discrimination learning: both the features alone and transfer compounds evoked some feature-specific responding that was reduced by feature extinction. But two aspects of the data suggested that discrimination learning also involved occasion setting. First, responding to the original compounds was substantially greater than responding to the features alone, and, second, feature extinction had little effect on responding to the original compounds.

Unlike previous experiments with operant SFP discriminations (Holland, 1995b), there was little evidence for transfer of occasion setting to the target of the other SFP discrimination. Thus, the results of Experiment 1 suggest that occasion setting with CS and US features may be functionally distinct, despite the similarity in the acquisition of the two discriminations.

\section{EXPERIMENT 2}

In Experiment 2, the apparent independence of occasion setting with CS and US features was further explored. Experiment 2 elaborated on Experiment 1 in three important ways. First, in Experiment 2 longer feature-target intervals and more salient target cues were used to encourage occasion setting and discourage simple feature conditioning (Holland, 1986, 1989; Holland \& Haas, 1993). One difficulty in the first transfer test in Experiment 1 was that the moderate levels of responding due to simple feature conditioning might have masked any transfer of occasion setting.

Second, in Experiment 2, transfer of occasion setting was examined not only when one feature was a CS and one feature was a US, but also when both features were CSs or both features were USs. Although considerable evidence shows transfer of occasion setting when both features are CSs (e.g., Holland, 1995b), there is little evidence to show that such transfer occurs when both fea- 
tures are USs. It is also possible that the parameters used in Experiment 1 (which differed slightly from the parameters normally used in our laboratory) might simply be parameters with which no transfer of occasion setting would occur, regardless of feature identity. Thus, the lack of transfer of occasion setting in Experiment 1 is meaningful only if transfer is shown both among those CS features and among those US features.

Finally, Experiment 2 included pseudodiscrimination control groups that received one SFP discrimination and one pseudodiscrimination $(F \rightarrow T+, T+$ ), which does not endow the feature with occasion-setting powers (Holland, 1991b). The pseudodiscrimination control groups controlled for the possibility that greater transfer of occasion setting with two CS, or two US, features, as opposed to one CS and one US feature, might reflect nothing more than greater confusion or generalization between two cues of the same modality.

\section{Method}

Subjects. The subjects were 48 female Sprague-Dawley rats obtained from Charles River (Raleigh, NC). At the beginning of the experiment, the rats were 120-150 days old and experimentally naive. They were maintained at $85 \%$ of their ad-lib body weights by measured feedings at the end of a session. Water was available at all times in their individual home cages, except as noted.

Apparatus. Four chambers were used in Experiment 2. They were similar to the chambers used in Experiment 1, with five exceptions: (1) The recessed food cups were centered on the front walls of the chambers, rather than slightly off center (the lever and chain were the same distance from the side wall as in the chambers used in Experiment 1). (2) A 6-W lamp was mounted on the door of each shell (door light), just above the center of one acrylic side wall of the chamber; when illuminated, this lamp served as one of the visual features. (3) The chambers were enclosed within only one sound-attenuating shell, rather than two. (4) The exhaust fans provided a continuous background noise of $72 \mathrm{~dB}$ rather than $68 \mathrm{~dB}$. And (5) there were two separate liquid dispensers (each operated by its own solenoid valve), rather than one.

Procedure. Initially, the rats were familiarized with the flavored (Kool-Aid) sucrose solution that served as the reinforcer. For half of the rats, this solution was grape flavor, and for the other half, it was orange. The solutions were made by dissolving $50 \mathrm{~g}$ of sucrose and $1 \mathrm{~g}$ of unsweetened Kool-Aid in 1 liter of tap water. On each of 3 days, approximately $5 \mathrm{ml}$ of orange or grape flavored solution (counterbalanced) was placed in a ceramic crucible in each rat's home cage. Each rat's water bottle was removed until all the Kool-Aid was consumed; at that time, the crucible was removed and the water bottle replaced.

Over the next 3 days, the rats were trained to drink from the food cup, press the lever, and pull the chain, as in Experiment 1 except that orange or grape Kool-Aid solution was used as the reinforcer. In all phases of Experiment 2, both responses were reinforced with only one flavored solution (orange or grape Kool-Aid, counterbalanced across groups). Next, the other solution (grape or orange) was placed in crucibles in the rat's home cage, as on the previous familiarization days. All rats consumed the second flavored solution rapidly on the first presentation, so no further familiarization was given.

The next 14 sessions were designed to place leverpressing and chainpulling under the control of the auditory target cues to be used later in SFP discrimination training. Each occurrence of one response was reinforced during presentations of a $79-\mathrm{dB}, 1500-\mathrm{Hz}$ square-wave tone, and each occurrence of the other response was reinforced during presentations of a $79-\mathrm{dB}$ white noise. In all sessions, tone and noise presentations were randomly intermingled. The response (leverpressing or chainpulling), the target (tone or noise), and reinforcer (orange or grape) were completely counterbalanced. The first three sessions were $30 \mathrm{~min}$ long and contained fifteen 30 -sec tone and fifteen $30-\sec$ noise presentations. In Sessions 4 and 5 , the cues were shortened to 15 and $10 \mathrm{sec}$, respectively. The session length was then extended to $40 \mathrm{~min}$ (Session 7), $45 \mathrm{~min}$ (Sessions 8 and 9), and $64 \mathrm{~min}$ (Session 10). Finally, Sessions 11-14 were 64 min long, and included eight 10sec tone and eight 10 -sec noise presentations. The subjects were then assigned to groups $(n s=8)$, maintaining complete counterbalancing of target, response, and reinforcer, and matching responding as closely as possible.

Serial feature-positive discrimination training was conducted over the next 21 sessions. Subjects in the experimental groups (Groups CS-CS, US-CS, and US-US) received training on two SFP discriminations. In Group CS-CS, two visual features (panel light and door light) were used; in Group US-CS, one Kool-Aid feature and one visual feature was used; and in Group US-US, two Kool-Aid features were used. In Group US-CS, the US feature was the same solution as that used for the reinforcer (grape or orange) and the CS feature was either the panel light or door light. In Group US-US, one of the features was the same flavored solution as that used for the reinforcer and the other feature was the other flavored solution. In each 64-min session, all subjects received four $\mathrm{F} 1-\mathrm{T} 1+$ trials (on which each $\mathrm{R} 1$ response during $\mathrm{T}$ 1 was reinforced), four T1 - trials (no reinforcement available), four F2-T2 + trials (on which each R2 response during T2 was reinforced), and four T2 - trials (no reinforcement available). All cues were $10 \mathrm{sec}$ in duration (except for US delivery, which was $0.5 \mathrm{sec}$ ), and the interval between feature onset and target onset was $30 \mathrm{sec}$ in both serial compounds. The identities of the feature cues were completely counterbalanced so that all featuretarget-response-reinforcer combinations were represented, except in Group US-CS, in which only 8 of the 16 possible combinations were represented.

Rats in the pseudodiscrimination (PD) control groups (Groups CS-PD, US/CS-PD, and US-PD) received training on one SFP discrimination and one pseudodiscrimination, in which responding was reinforced on both feature $\rightarrow$ target and target-alone trials. The rats in the pseudodiscrimination control groups received training identical to that received by their corresponding experimental group, except that R2 responding was reinforced on both F2-T2 and T2 trials. Counterbalancing of feature/pseudofeature, target, and response was as complete as possible. The three pseudodiscrimination control groups were run separately from the three experimental groups; however, trial sequences, ITIs, and all aspects of training and testing (except for the reinforcement of R2 during $\mathrm{T} 2$ trials) were identical to those received by their corresponding experimental groups.

Next, the rats received two test sessions to evaluate transfer. In each 64-min session, there were two presentations of each of eight trial types. The eight trial types included the two features alone ( $\mathrm{F} 1$ and $\mathrm{F} 2$ ), the two targets alone ( $\mathrm{T} 1$ and $\mathrm{T} 2$ ), the two original serial compounds ( $\mathrm{F} 1-\mathrm{T} 1$ and $\mathrm{F} 2-\mathrm{T} 2$ ), and, of most interest, the two novel transfer compounds (F1-T2 and F2-T1). Responding was not reinforced during the two test sessions.

The effects of feature extinction were then evaluated. The rats in the three experimental groups first received seven sessions in which one feature was extinguished while the other SFP discrimination was retrained. In each 64-min session, half of the rats in each of these groups received eight F1-alone trials, four F2-T2+ trials, and four T2 - trials; the other half received eight F2-alone trials, four F1-T1 + trials, and four T1 - trials. During these sessions, half of the rats in Groups US-PD and CS-PD received feature extinction $(\mathrm{Fl}-)$ and pseudodiscrimination retraining $(\mathrm{F} 2-\mathrm{T} 2+$ and $\mathrm{T} 2+)$ trials, and the other half received pseudofea- 
ture extinction (F2 - ) and SFP discrimination retraining (F1-T1 + and $\mathrm{T} 1-$ ) trials. Since there were too few rats in Group US/CS$\mathrm{PD}$ to permit a well-counterbalanced examination of feature extinction, that group was not included in this evaluation.

All rats in the remaining five groups then received two postextinction test sessions, identical to the test sessions described previously.

Finally, the rats in Group US-US received a single retraining session, identical to the previous discrimination training sessions, followed by a final test session. This final test session was similar to previous test sessions except that all Kool-Aid was removed from the dispensers; its purpose was to determine whether the effective feature was the flavored sucrose or any auditory cues provided by the operation of the solenoid valves.

\section{Results and Discussion}

During initial training, the rats learned to perform one response during one target cue and a different response during the other target cue. By the final session, both leverpressing and chainpulling occurred at similar levels during the tone or noise target cues. Across all six groups, the rats made at least one correct response on $92 \%$ of the trials and at least one incorrect response on $23.5 \%$ of the trials.

Discrimination training. Figure 4 shows the results of discrimination training in all six groups in terms of difference scores (percentage of trials with a response on feature-target or pseudofeature-target trials minus percentage of trials with a response on target-alone trials). In general, the experimental groups acquired both SFP discriminations at similar rates, regardless of the counterbalanced factors of feature, target, response, and reinforcer. However, during the first four sessions, 7 of the 8 rats in Group US-US responded more during compounds with the noise $(85.2 \%)$ than they did during those with the tone $(56.2 \%)$. Also in those sessions, in Group USUS, responding to the serial compound that included the novel US (60.9\%) was numerically lower than responding to the serial compound that included the US that was used as the reinforcer $(80.4 \%)$, although that pattern was shown by only 5 of 8 rats. By the end of training, however, rats in the experimental groups responded at equal rates on all kinds of compound trials. The control groups that received one SFP and one PD showed a similar pattern on SFP trials; however, responding occurred at high levels on both PD compound and PD element-alone trials, regardless of the counterbalancing factors.

Test 1. Figures 5-7 show the results of Test 1 . Because there were no effects of any of the counterbalancing factors, including whether the feature was a CS or a US in Groups US-CS and US-CS/PD, the data are collapsed across all of those factors. Consider first the performance of the experimental groups that received two SFPs (Figure 5). Performance on the original discriminations was maintained. In all three groups, the rats showed significantly more $R 1$ responding on F1-T1 trials than on $T 1$ trials $[T s(8)=0]$ and significantly more $\mathrm{R} 2$ responding on $\mathrm{F} 2-\mathrm{T} 2$ trials than on T2 trials $[T \mathrm{~s}(8) \leq 3.5]$. Notably, neither feature controlled much responding when presented alone-significantly less

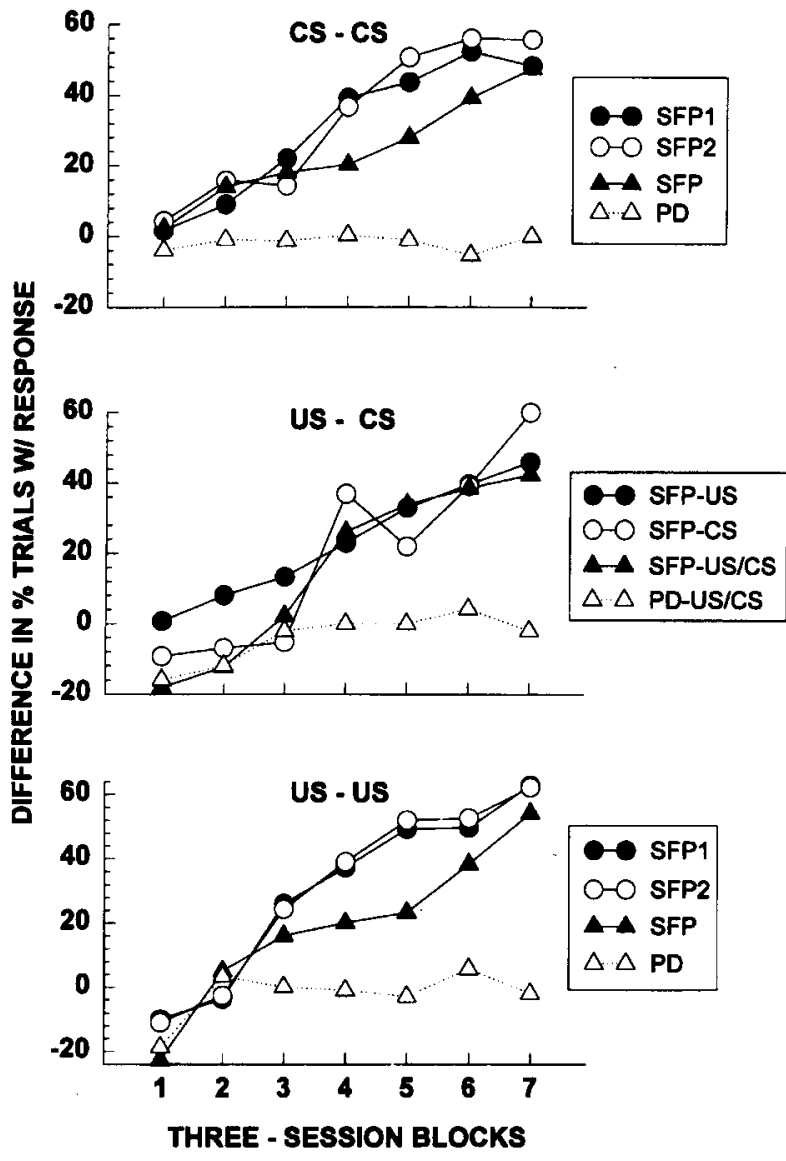

Figure 4. Acquisition of serial feature positive (SFP) discrimination or pseudodiscrimination (PD) performance in Experiment 2. The top panel shows responding in the groups for which both features were visual cues (Groups CS-CS and CS-PD); the middle panel shows responding in the groups for which one feature was a flavored liquid and one was a visual cue (Groups US-CS and US/CS-PD); the bottom panel shows responding in the groups for which both features were flavored liquids (Groups US-US and US-PD). The response measure shown is the mean difference between the percentage of trials on which a targetappropriate response occurred on serial compound trials and on target-alone trials. In the top and bottom panels, the circles indicate responding on the two SFP discriminations (SFP1 and SFP2) in the experimental groups, and the triangles indicate responding on the one SFP discrimination (SFP) and the one pseudodiscrimination (PD) in the control groups. In the center panel, the circles indicate responding on the SFP discrimination involving the flavored sucrose feature (SFP-US) or the discrimination that involved the visual feature (SFP-CS) in Group US-CS, and the triangles indicate responding on the SPF discrimination, combined across the two feature types (SFP-US/CS), or the pseudodiscrimination, combined across the two pseudofeature types (PD-US/CS), in Group US/CS-PD. US = flavored sucrose feature; $\mathrm{CS}=$ visual feature.

than was responding on the original compound trials $[T \mathrm{~s}(8)=0]$. Hence, our efforts to minimize feature conditioning were apparently successful.

Of greater interest, however, was responding during the two novel transfer compounds (F1-T2 and F2-T1). In Groups CS-CS and US-US, substantial transfer of 

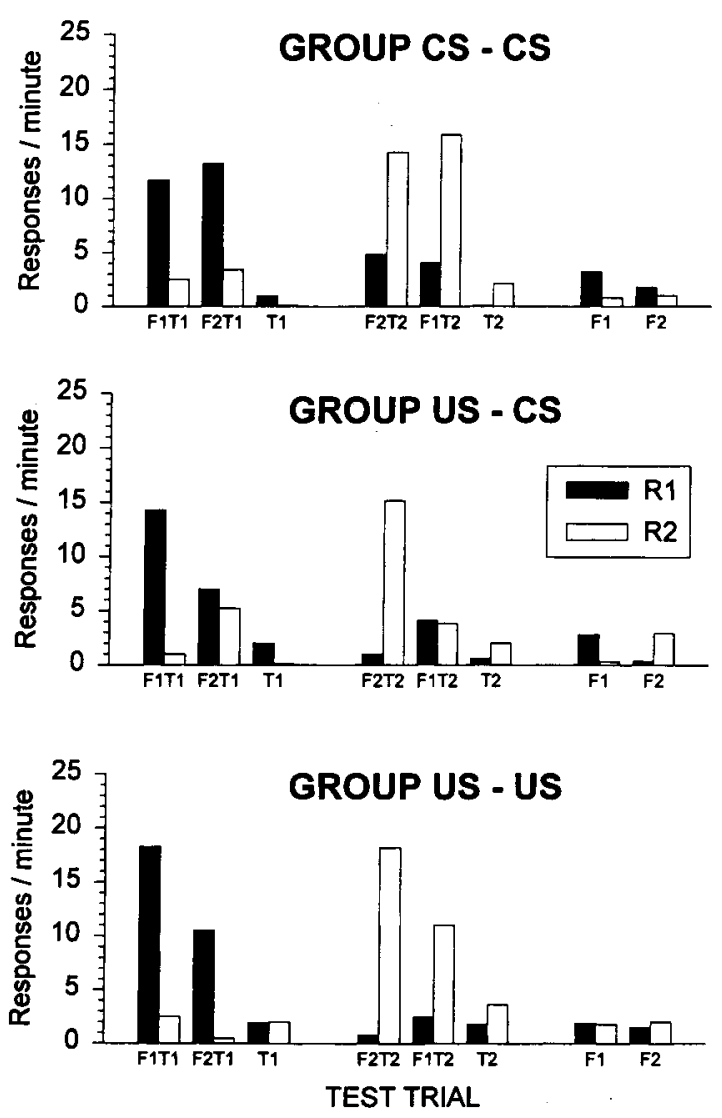

Figure 5. Mean response rates in Groups CS-CS, US-CS, and US-US during Test 1 in Experiment 2. In training, R1 (leverpressing or chainpulling, counterbalanced) was reinforced during the target on F1-T1 compound trials, and R2 was reinforced on F2-T2 compound trials. Responding on feature-alone trials (F1 and F2) was measured at the time when a target would normally have been presented. $F 1$ and $F 2$ were two visual cues, counterbalanced, in Group CS-CS; two flavored sucrose solutions, counterbalanced, in Group US-US; and one of two flavored solutions and one of two visual cues, counterbalanced, in Group US-CS. T1 and T2 were two auditory target cues, counterbalanced. $\mathrm{US}=$ flavored sucrose feature; $\mathrm{CS}=$ visual feature.

occasion setting was observed. Both Group CS-CS and Group US-US showed significantly more target-specific than feature-specific responding on F1-T2 and F2-T1 trials $[T \mathrm{~s}(8) \leq 1.5]$ and more target-specific responding on transfer compound trials than on feature-alone or target-alone trials $(T \mathrm{~s}=0)$. Indeed, in Group CS-CS, transfer was complete: target-specific responding on F1-T1 and F2-T1 trials did not differ $[T(7)=15]$, nor did responding on $\mathrm{F} 2-\mathrm{T} 2$ and $\mathrm{F} 1-\mathrm{T} 2$ trials $[T(8)=13]$. In Group US-US, although large, transfer was not complete; target-specific responding was greater on F1-T1 trials than on F2-T1 trials $[T(7)=1]$ and responding was greater on F2-T2 trials than on F1-T2 trials $[T(7)=4]$. Thus, transfer of occasion setting was shown when both features were CSs or when both were USs.

In Group US-CS, however, there was only minimal evidence of transfer of occasion setting. First, target-specific responding was not reliably more frequent than feature- specific responding on either F1-T2 $[T(8)=12.5]$ or $\mathrm{F} 2-$ $\mathrm{T} 1[T(7)=8]$ trials. Second, although the rats in Group US-CS showed significantly more target-specific (R1) responding on F2-T1 than on $\mathrm{T} 1$-alone trials $[T(8)=0]$, target-specific (R2) behavior on F1-T2 trials did not differ from that on T2-alone trials $[T(8)=21]$. Furthermore, target responding was more frequent on original compound trials than on transfer compound trials $[T \mathrm{~s}(8)=0]$.

Figure 6 shows the performance of the pseudodiscrimination control groups that received one SFP and one pseudodiscrimination. Performance on the original SFP discrimination was maintained; in all three groups, the rats showed significantly more $\mathrm{R} 1$ responding on $\mathrm{F} 1$ $\mathrm{T} 1$ than on $\mathrm{T} 1$ trials $[T \mathrm{~s}(8)=0]$. As in the experimental groups, there was little evidence of conditioning to the F1 feature; responding on $F 1$ trials was reliably less than responding on $\mathrm{F} 1-\mathrm{T} 1$ trials $[T \mathrm{~s}(8)=0]$. Performance on
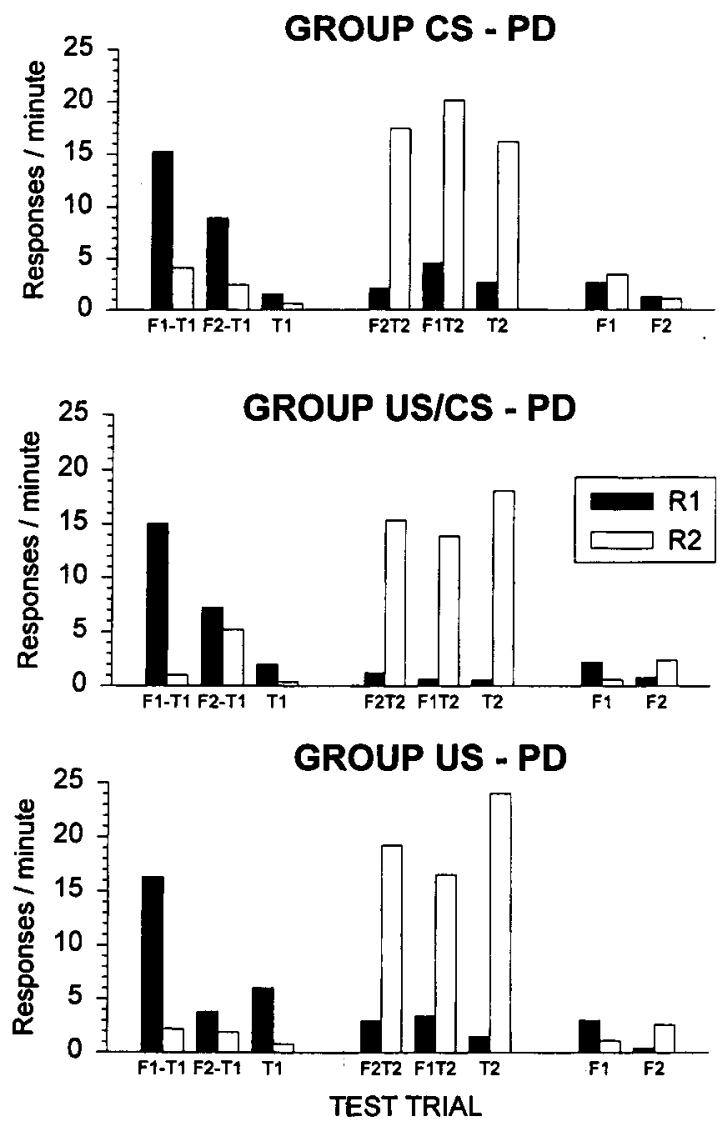

Figure 6. Mean response rates of rats in Groups CS-PD, US/CS-PD, and US-PD during Test 1 in Experiment 2. In training, $\mathrm{R1}$ (leverpressing or chainpulling, counterbalanced) was reinforced during the target on F1-T1 compound trials, and R2 was reinforced on $F 2-T 2$ compound and T2-alone trials. Responding on feature-alone trials (F1 and F2) was measured at the time when a target would normally have been presented. F1 and F2 were two visual cues, counterbalanced, in Group CS-PD; two flavored sucrose solutions, counterbalanced, in Group US-PD; and one of two flavored solutions and one of two visual cues, counterbalanced, in Group US/CS-PD. T1 and T2 were two auditory target cues, counterbalanced. US = flavored sucrose feature; $\mathrm{CS}=$ visual feature; $\mathrm{PD}=$ pseudodiscrimination. 
the pseuododiscrimination, however, differed from that on the SFP discrimination. Specifically, R2 responding on F2-T2 and T2 trials did not differ reliably in Groups CS-PD $[T(8)=13]$ or US/CS-PD $[T(8)=14]$, and in Group US-PD, R2 responding was significantly greater on $\mathrm{T} 2$ trials than on $\mathrm{F} 2-\mathrm{T} 2$ trials $[T(7)=2]$. Responding on both $\mathrm{F} 2-\mathrm{T} 2$ and $\mathrm{T} 2$-alone trials was greater than responding on pseudofeature (F2) alone trials $(T \mathrm{~s}=0)$.

More importantly, Groups US-PD and US/CS-PD showed little feature generalization. In agreement with previous data (Holland, 1991b), the F2 pseudofeature failed to enhance responding to the target of the SFP discrimination: Target-specific responding on F2-T1 trials was not reliably greater than that during $\mathrm{T} 1$-alone trials $[T \mathrm{~s}(8)=16$ and 6.5 in those two groups, respectively $]$. Similarly, the F1 feature failed to enhance responding to the target of the pseudodiscrimination in those groups: Target-specific responding was not reliably greater on F1-T2 trials than on $T 2$ trials $(T s \geq 16)$. Some feature generalization, however, was observed in Group CS-PD: Target-specific responding during F2-T1 trials was reliably greater than during $T 1$ trials $[T(8)=0]$.

Figure 7 summarizes the performance of experimental and control groups in Test 1. Each bar reflects the difference between target-specific responding on compound and target-alone trials. For the experimental groups, the open bars show the difference scores for the two original discriminations (combined). All the experimental groups showed similar performance on the original discriminations $\left[U_{\mathrm{s}}(8,8) \geq 24.5\right]$. However, transfer difference scores (filled bars) were significantly smaller in Group US-CS than in Groups CS-CS $[U(8,8)=6.5]$ and US-US $[U(8,8)=11.5]$, which did not differ reliably from each other $[U(8,8)=18]$. Thus, transfer of occasion setting was greater when both features were CSs or USs than when one feature was a US and one was a CS.

In the pseudodiscrimination control groups, the open bars in Figure 7 show the difference scores for the original SFP discriminations. All the pseudodiscrimination groups showed similar performance on the original SFP discrimination $(U s \geq 21.5)$. The hatched bars show the difference in responding on F2-T1 and T1 trials, that is, the effect of the pseudofeature on the target of the SFP discrimination. Those difference scores were reliably greater in Group CS-PD than in Group US-PD $[U(8,8)=$ $11.5]$; no other between-group difference was significant $\left(U_{\mathrm{s}} \geq 18\right)$.

The primary purpose of the three pseudodiscrimination groups was to permit the evaluation of transfer in the experimental groups, beyond simple generalization between the features, and any factors apart from the SFP training contingencies. To this end, we compared transfer difference scores of each experimental group (solid bars in Figure 7) with the F2-T1 difference scores in its partner pseudodiscrimination group (hatched bars). Transfer was reliably greater in Groups CS-CS and US-US than in Groups CS-PD $[U(8,8)=13]$ and US-PD $[U(8,8)=$ $1.5]$, respectively. Thus, the transfer observed in Groups CS-CS and US-US was significantly greater than that attributable to simple feature generalization. In contrast,

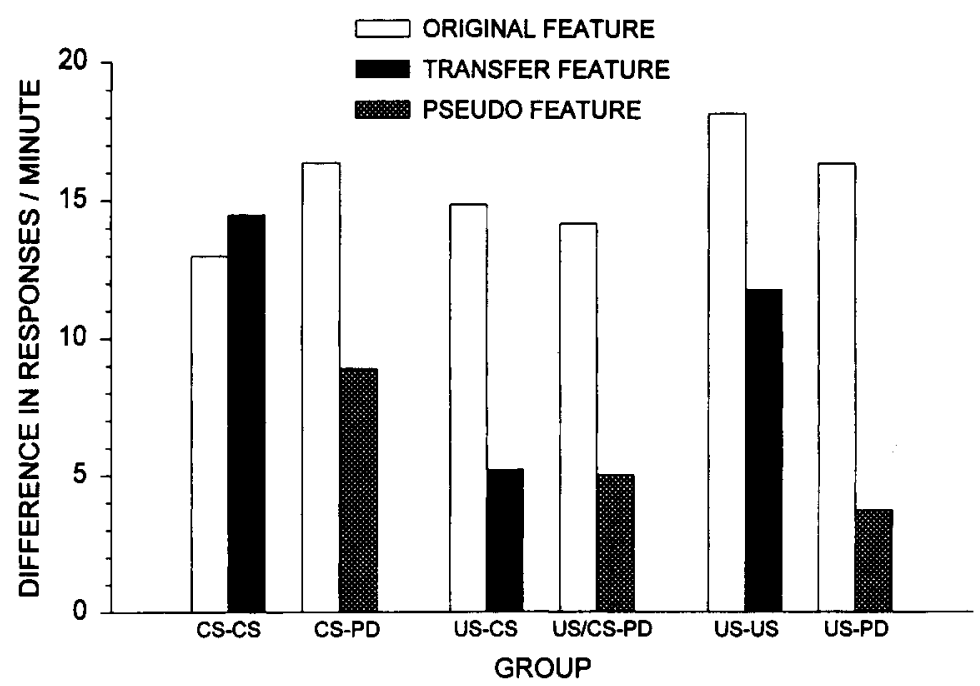

Figure 7. Discrimination difference scores in Test 1 of Experiment 2. Each bar represents the mean difference between the rate of target-appropriate responding on serial compound trials and target-alone trials. The open bars signify performance on the original serial feature-positive discrimination(s), the solid bars indicate performance on transfer discriminations in which the features and targets not originally trained together were combined (Groups CS-CS, US-CS, and US-US only), and the hatched bars show performance when the compounds included the pseudofeature and the target from the original serial feature-positive discrimination (Groups US-PD, US/CS-PD, and CSPD only). US = flavored sucrose feature; $C S=$ visual feature; $P D=$ pseudodiscrimination. 
the transfer difference scores did not differ reliably between Groups US-CS and US/CS-PD $[U(8,8)=32]$; thus, even the small amount of apparent transfer seen in Group US-CS was not attributable to true transfer of occasion setting.

Test 2. Figure 8 shows performance during Test 2 , which was identical to Test 1 but occurred after extinction of one feature or pseudofeature. For ease of comparison with the major results of Test 1 , Figure 8 shows only the discrimination difference scores for target-specific responding, as in Figure 7.

With the exception of pseudodiscrimination responding in Group CS-PD, performance in Test 2 was depressed

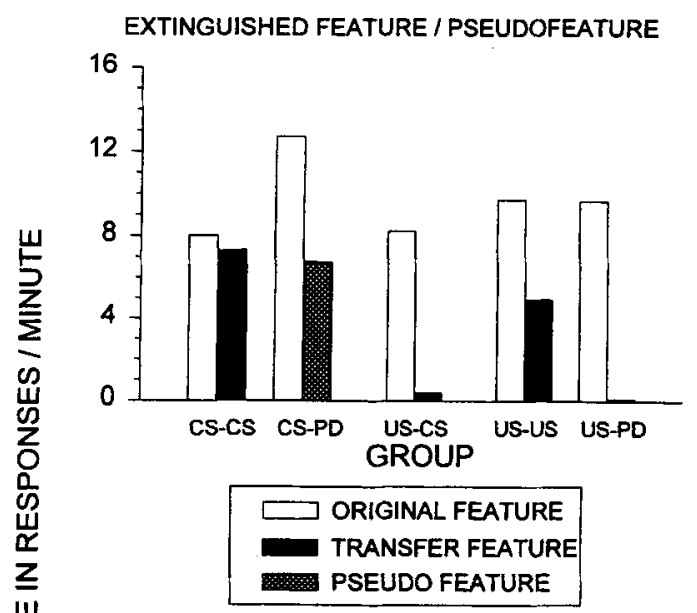

NONEXTINGUISHED FEATURE / PSEUDOFEATURE

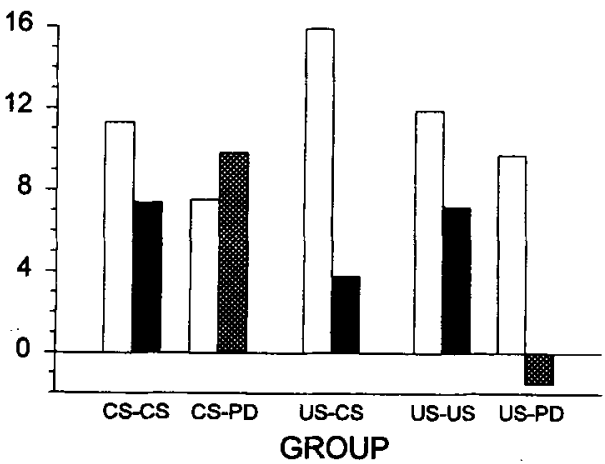

Figure 8. Discrimination difference scores in Test 2 of Experiment 2, after extinction of one of the two features in the three experimental groups, and of either the feature or the pseudodiscrimination feature in the two control groups. Performance involving the extinguished feature or pseudofeature is shown in the top panel, and responding involving the nonextinguished feature or pseudofeature is shown in the bottom panel. Each bar represents the mean difference between the rate of targetappropriate responding on serial compound trials and targetalone trials. The open bars signify performance on the original feature-positive discrimination(s), the solid bars indicate performance on transfer discriminations in which the features and targets not originally trained together were combined (Groups CS-CS, US-CS, and US-US only), and the hatched bars show performance when the compounds included the pseudofeature and the target from the original serial feature-positive discrimination (Groups US-PD and CS-PD only). US = flavored sucrose feature; $\mathbf{C S}=$ visual feature; $\mathbf{P D}=$ pseudodiscrimination. relative to that in Test 1 (compare Figures 7 and 8 ). Nevertheless, reliable performance on the original discriminations (open bars) was preserved, with both extinguished (top panel) and nonextinguished (bottom panel) features. In all five groups, the original discrimination difference scores were reliably greater than zero, that is, responding was greater on compound than on targetalone trials $[T s(8)=0]$. Furthermore, transfer performance (filled bars) was preserved in Groups CS-CS and US-US, with both extinguished and nonextinguished features $[T \mathrm{~s}(8) \leq 1.5]$. Also, as in Test 1 , there was little evidence of transfer in Group US-CS [ $T(8)=12]$. Thus, the major patterns of responding shown by the three discrimination groups in Test 1 were maintained after extinction of one feature cue, supporting our interpretation of that responding as reflecting occasion setting. For Groups CS-CS and US-US, this claim is further supported by the observation that the original discrimination difference scores did not differ as a function of whether the feature was extinguished (top panel) or not (bottom panel) $\left[T_{\mathrm{s}}(8) \geq 8\right]$. In contrast, in Group US-CS, despite the preservation of performance on both original discriminations, there were selective effects of feature extinction: the original discrimination difference scores were reliably lower with the extinguished feature than with the nonextinguished feature $[T(8)=2.5]$.

As in Test 1, with US features transfer responding to the target of an SFP discrimination was greater when it was preceded by another feature (Group US-US, filled bars) than when it was preceded by a pseudofeature (Group US-PD, hatched bars), both in subjects that received extinction of those cues and in subjects that did not $[U s(8,4)=4]$. In Group US-PD, performance on the original SFP discrimination with extinguished and nonextinguished features did not differ $[U(4,4)=2.5]$. Furthermore, the pseudofeature did not enhance responding to $T 1$ (hatched bars) $[T(7)=16.5]$, nor did that responding differ as a function of pseudofeature extinction $[U(4,4)=5]$. Thus, as in Test 1 , the transfer of occasion setting observed in Group US-US was not solely attributable to simple stimulus generalization between the features.

In contrast, with CS features, the differences between discrimination and pseudodiscrimination performance found in Test 1 were not generally maintained, primarily because pseudodiscrimination responding in Group CSPD was maintained at the same high level as was observed in Test 1, while discrimination responding was depressed (compare Figures 7 and 8). Neither the original nor the transfer discrimination difference scores in Group CS-CS differed reliably from the pseudodiscrimination transfer scores in Group CS-PD, regardless of whether the feature or pseudofeature was extinguished $[U s(8,4) \geq 7.5]$ or not $[U s(8,4) \geq 9]$. Furthermore, within Group CS-PD, performance with the nonextinguished feature did not differ from that with the nonextinguished pseudofeature $[U(4,4)=7]$, although performance with the extinguished feature was reliably greater than that with the extinguished pseudofeature $[U(4,4)=1]$. We 
have no account for the high levels of responding observed with the pseudofeature in Group CS-PD in Test 2.

Test 3. After one retraining session, Group US-US received a final test session with all Kool-Aid removed from the dispensers. Target-appropriate responding during the targets alone, original compounds, and transfer compounds was $2.4,3.0$, and 3.2 responses $/ \mathrm{min}$, respectively. Thus, Test 3 showed that properties of the KoolAid solutions themselves, rather than the auditory cues accompanying the operation of the dispensers, was critical to their occasion-setting powers.

\section{GENERAL DISCUSSION}

In two experiments, rats' acquisition of two operant SFP discriminations was similar regardless of whether the features were visual cues or sucrose solutions. Occasion setting readily transferred when the features were two visual cues or two sucrose solutions. However, we observed no transfer of occasion setting when one feature was a visual cue and one feature was a sucrose solution, regardless of whether the features evoked significant levels of feature-specific responding (Experiment 1) or not (Experiment 2). Furthermore, the pseudodiscrimination control groups in Experiment 2 showed that greater transfer of occasion setting with same-modality features was not due solely to feature generalization.

These experiments do not identify what properties of the CS and US features were responsible for the rats' failure to exhibit transfer across those two kinds of features. Nevertheless, several differences between the US and CS features are worth mentioning. First, the critical difference may be that implied by our terminology: features with substantial intrinsic reinforcing or motivational powers may be treated quite differently from those without. Second, although particular appetitive and consummatory behaviors (e.g., cup entry and licking) were required for an animal to receive a US feature, no particular response requirement existed for receipt of a CS feature. Third, because in Experiment 1 the US feature also served as the reinforcer, it was presented outside of its conditional relation in a way that a CS feature was not. (In this regard, however, it should be recalled that transfer was substantial in Group US-US of Experiment 2 , in which one of the US features was used as the reinforcer and the other was not; thus, this difference alone was insufficient to prevent transfer.) Future studies might deliver the flavored sucrose features independently of behavior (e.g., through implanted cannulas) or use a different reinforcer (such as a food pellet) while maintaining the same two flavored sucrose solutions as features. Notably, however, whatever was responsible for the rats' failure to treat US and CS features as equivalent in transfer tests did not significantly affect the rate of original discrimination learning.

Regardless of the categorization principle that distinguishes a US and CS feature, it is clear that these features failed to exhibit mutual transfer, despite robust transfer within each modality. This result constrains ac- counts of the frequent observation of greater transfer of occasion setting to targets of other occasion setters than to targets with other training histories (Holland, 1989, 1995b; Lamarre \& Holland, 1987; Rescorla, 1985).

Lamarre and Holland (1987) offered two accounts for the dependence of transfer on the training history of target cues. The first account postulated the existence of two memory systems, one representing events in simple associative relations and another representing events in more complex, hierarchical relations, such as occasion setting. Transfer might occur more readily within, than between, systems. To accommodate the present results, the hierarchical memory system might be subdivided so that visual CS and flavor US features are represented in different modules. But, as Holland and Coldwell (1993) noted, the two-memory system notion loses its appeal as post hoc strictures are added.

Alternately, Lamarre and Holland (1987) speculated that comparable training treatments render the targets of occasion setters (and/or the occasion setters themselves) more similar, and hence those events acquire functional equivalence. Recent data support this view. Bonardi and Hall (1994) showed that when an event previously trained as a target in an SFP discrimination was conditioned, more generalized responding was observed to the target of another SFP discrimination than to another cue that was not so trained. However, it would be awkward to attribute the transfer observed in Groups CS-CS and USUS of Experiment 2 to such enhanced generalization between target cues, because the two target cues controlled qualitatively different operant responses in the transfer tests (see also Holland \& Coldwell, 1993).

The pattern of transfer observed in Groups CS-CS and US-US is consistent, however, with enhanced generalization between the feature cues. This possibility is supported by Honey and Hall's (1989) finding that events trained with common consequents also come to generalize more to each other. Nevertheless, the lack of transfer across CS and US features observed in both experiments reported here demands that, within this acquired equivalence perspective, a limit be placed on the extent to which comparable training histories can overcome perceptual differences between cues. Perhaps transfer is limited to events already possessing certain sets of shared attributes (e.g., Jenkins, 1985). Although previous studies showed substantial transfer of occasion setting between visual and auditory features (Holland, 1995b), those cues might share more features than the CS and US features used here.

These results also suggest modifications to a real-time network model of configural learning recently adapted to successfully describe much of the data from occasionsetting experiments (Schmajuk, Lamoureux, \& Holland, 1994). That model redescribes occasion setting in configural terms, representing those configural cues as hidden units, each with inputs from a variety of sensory units and outputs to a variety of response units. In addition, learned responding is affected by direct, nonconfigural connections between sensory and response units. 
Within this model, substantial transfer occurs to targets of other SFP discriminations because the various feature and target cues all share connections with a common pool of configural units. In contrast, little transfer occurs to separately trained cues: those cues primarily activate response units directly and hence fail to activate the configural units when presented in compound with a feature cue. Our failure to observe transfer between visual and flavor cues suggests that not all cues share connections with the same pool of configural hidden units. Cues as disparate as those from visual and gustatory modalities might simply share fewer of those units.

Whatever their ultimate explanation, the results clearly show differences in the processing of visual CS and flavor US features in operant serial feature-positive discrimination learning. Thus, transfer of occasion setting is affected not only by the training contingencies but also by the intrinsic properties of the events involved in those contingencies.

\section{REFERENCES}

Bonardi, C., \& Hall, G. (1994). Occasion-setting training renders stimuli more similar: Acquired equivalence between the targets of feature positive discriminations. Quarterly Journal of Experimental Psychology, 47B, 63-82.

BottJer, S. W., \& Hearst, E. (1979). Food delivery as a conditional stimulus: Feature learning and memory in pigeons. Journal of the Experimental Analysis of Behavior, 31, 189-207.

CAPALDI, E. J. (1967). A sequential hypothesis of instrumental learning. In K. W. Spence \& J. T. Spence (Eds.), The psychology of learning and motivation (Vol. 1, pp. 67-156). New York: Academic Press.

GarCIA, J. (1989). Food for Tolman: Cognition and cathexis in context. In T. Archer \& L.-G. Nilsson (Eds.), Aversion, avoidance, and anxiety: Perspectives on aversively motivated behavior ( $\mathrm{pp} .45-85$ ). Hillsdale, NJ: Erlbaum.

Goddard, M. J., Holland, M., O'Brien, J., \& Hansen, D. (1994). Improving conditioning with unsignaled, but not signaled, unconditioned stimuli. Learning \& Motivation, 25, 288-312.

Goddard, M. J., \& JeNKINS, H. M. (1988). Blocking of a CS-US association by a US-US association. Journal of Experimental Psychology: Animal Behavior Processes, 14, 177-186.

Holland, P. C. (1983). Occasion-setting in Pavlovian feature positive discriminations. In M. L. Commons, R. J. Herrnstein, \& A. R. Wagner (Eds.), Quantitative analyses of behavior: Discrimination processes (Vol. 4, pp. 183-206). New York: Ballinger.

Holland, P. C. (1986). Temporal determinants of occasion setting in feature positive discriminations. Animal Learning \& Behavior, 14, 111-120.

Holland, P. C. (1989). Occasion setting with simultaneous compounds in rats. Journal of Experimental Psychology: Animal Behavior Processes, 15, 183-193.

Holland, P. C. (1991a). Acquisition and transfer of occasion setting in operant feature positive and feature negative discriminations. Learning \& Motivation, 22, 366-387.

Holland, P. C. (1991b). Transfer of control in ambiguous discrimi- nations. Journal of Experimental Psychology: Animal Behavior Processes, 17, 231-248.

Holland, P. C. (1992). Occasion setting in Pavlovian conditioning. In D. Medin (Ed.), The psychology of learning and motivation (Vol. 28, pp. 69-125). San Diego, CA: Academic Press.

Holland, P. C. (1995a). The effects of intertrial and feature-target intervals on operant serial feature-positive discrimination learning. Animal Learning \& Behavior, 23, 411-428.

HollaNd, P. C. (1995b). Transfer of occasion setting across stimulus and response in operant feature positive discriminations. Learning \& Motivation, 26, 239-263.

Holland, P. C., \& Coldwell, S. E. (1993). Transfer of stimulus control in operant feature-negative discriminations. Learning \& Motivation, 24, 345-375.

Holland, P. C., \& Forbes, D. T. (1982). Control of conditional discrimination performance by CS-evoked event representations. $A n$ imal Learning \& Behavior, 10, 249-256.

Holland, P. C., \& HaAs, M. L. (1993). The effects of target salience in operant feature positive discriminations. Learning \& Motivation, 24, 119-140.

Holz, W. C., \& Azrin, N. H. (1961). Discriminative properties of punishment. Journal of the Experimental Analysis of Behavior, 5, 229234.

Honey, R. C., \& Hall, G. (1989). The acquired equivalence and distinctiveness of cues. Journal of Experimental Psychology: Animal Behavior Processes, 15, 338-346.

JENKINS, H. M. (1985). Conditioned inhibition of key pecking in the pigeon. In R. R. Miller \& N. E. Spear (Eds.), Information processing in animals: Conditioned inhibition (pp. 327-353). Hillsdale, $\mathrm{NJ}$ : Erlbaum.

KoNORSKI, J., \& LAWICKA, W. (1959). Physiological mechanism of delayed reactions: $I$. The analysis and classification of delayed reactions. Acta Biologiae Experimentalis, 19, 175-197.

Lamarre, J., \& Holland, P. C. (1987). Acquisition and transfer of serial feature negative discriminations. Learning \& Motivation, 18, 319-342.

ReberG, D., \& Memmotт, J. (1979). Shock as a signal for shock or no-shock: A feature-negative effect in conditioned suppression. Journal of the Experimental Analysis of Behavior, 32, 387-397.

Rescorla, R. A. (1985). Conditioned inhibition and facilitation. In R. R. Miller \& N. E. Spear (Eds.), Information processing in animals: Conditioned inhibition (pp. 299-326). Hillsdale, NJ: Erlbaum.

Schmajuk, N. A., LamoureuX, J., \& Holland, P. C. (1994, November). Occasion setting and stimulus configuration: A neural network approach. Paper presented at the annual meeting of the Psychonomic Society, St. Louis.

SOLOMON, R. L. (1977). An opponent-process theory of motivation: V. Affective dynamics of eating. In L. M. Barker, M. R. Best, \& M. Domjan (Eds.), Learning mechanisms in food selection (pp. 255269). Waco, TX: Baylor University Press.

STADDON, J. E. R., \& INNIS, N. K. (1969). Reinforcement omission on fixed-interval schedules. Journal of the Experimental Analysis of Behavior, 12, 689-700.

TERRY, W. S., \& WAGNER, A. R. (1975). Short-term memory for "surprising" versus "expected" unconditioned stimuli in Pavlovian conditioning. Journal of Experimental Psychology: Animal Behavior Processes, 1, 122-133.

(Manuscript received June 17, 1995; revision accepted for publication July 31,1995 .) 